\title{
Determining High Safety Risk Scenarios by Applying Context Information
}

\author{
Stewart Worrall, David Orchansky, Favio Masson, Juan Nieto and Eduardo Nebot
}

\begin{abstract}
When mining vehicle operators take risks, there is a increased probability of an accident that can cause injuries, fatalities and large financial costs to the mine operators. It can be assumed that the operators do not intentially take unnecessarily high risk, and that the risks are hidden due to factors such as adverse weather, fatigue, visual obstructions, boredom, etc. This paper examines the potential of measuring the risk of danger in a multi-agent situation by using the safe rules of operation defined by mining safety management.

The problem with measuring safety is that the safe rules of operation are heavily dependent on the context of the situation. What is considered normal practice and safe in one part of the mine (such as performing a u-turn in a parking lot) is not safe on a haul road. To be able to measure safety, it is therefore necessary to understand the different context areas in a mine so that feedback of unsafe behaviour presented to the operator is relevant to the context of the situation. This paper presents a novel method for generating context area information using the vehicle trajectory information collected from a group of vehicles interacting in an area. Results are presented using real-life data collected from several operating fleets of mining vehicles. The algorithms presented have potential application to a large variety of environments including Intelligent Transportation Systems (ITS).
\end{abstract}

Index Terms-Vehicle Safety, Collision Avoidance, Context, Data Mining.

\section{INTRODUCTION}

$\mathbf{E}$ $\mathrm{ACH}$ year there are hundreds of mine haulage accidents that result in significant number of deaths, lost-time injuries and large financial costs due to machine downtime and the repair of equipment. The majority of these accidents occur as a result of a human operator failing to comprehend the risks involved when operating of the vehicle. This paper examines how high risk situations can be detected using rules of safe operation, and the importance of context in determining the level of risk. This allows feedback to be provided to the vehicle operator in the event a high risk situation, enabling the operator to alter their actions to reduce the risk.

An automated process for determining important context locations in a mine is presented, with results provided using real data collected from mining operations. This paper mainly refers to mining operations but the algorithms derived have application to all areas where diverse type of mobile agent interact within shared areas. These includes industrial applications such as stevedoring and construction where mixture of

Stewart Worrall, David Orchansky, Juan Nieto and Eduardo Nebot are with the Australian Center for Field Robotics at the University of Sydney. Favio Masson is with the Universidad Nacional del Sur. E-mail: s.worrall@acfr.usyd.edu.au vehicles and people operate in a confined environment. It is also applicable to the Intelligent Transportation System area where we have high density of different type of agents such as vehicles, bikes, people operating in restricted areas such as road, intersections and parking lot.



Fig. 1. (a) Dusty or (b) foggy conditions make it difficult for a vehicle operator to determine how close they are travelling to other vehicles.

Section II presents current research in this area, which focuses primarily on determining the likelihood of an collision occuring and warning the operator when the threat of collision is above some threshold. The problem with existing approaches is that it is often not possible to determine whether a situation will result in a collision with sufficient certainty (to minimise false alarms) until it is almost too late to avoid. This is considered to be a form of reactive feedback, and 
it requires the operator to react quickly when an imminent collision is detected. An examples is presented in [1] where different level of warning are generated according to the proximity of the center or side of the road. Other approaches requires the sensing of location of other vehicles in proximity to generate some sort of alarm when some level of collision risk is evaluated. Furthermore, significant research to reduce false alarms has been done estimating roads and probabilistic methods to estimate time to collision with the evaluated roads [2], [3] [4].

An alternate approach presented in this paper is to provide preventitive feedback to the operators, meaning that the operator is warned when the risk of collision is considered high based on a predetermined set of safety rules. In the case of a mining environment, this includes rules governing minimum safe distances between vehicles, and speed limits for different areas of the mine. The problem is that the rules of safe operation change depending on the context of the situation. Context is a term introduced here to refer to the high level meaning of the situation that alters the rules used to determine risk. This can include concepts such as the semantics of the location, weather conditions, level of fatigue and many others. In a mining environment for example, vehicles on haul roads are required to maintain particular distance of separation which is greater than if they are located in a parking area or refuelling bay. In this example, a haul road is a different context area to the parking area and the refuelling bay. The paper focuses on the role of context areas in changing the rules of safe operation for a vehicle.

A digital map is required to determine the location of the roads and context areas. Determining the location of context areas can be done manually, though this is a time consuming process requiring skilled operators that know and understand the area very well. The mining environment presents a particular challenge for generating map information due to the dynamic nature of mining operations. Roads and open areas are constantly created and removed as the areas being mined change. These creates additional challenges to the operator since the environment and roads are changing all the time.

Sections III to V describe a novel technique for generating context area information by processing collected vehicle position information. Section III describes a system that automatically logs and collects vehicle trajectory information from a fleet of vehicles. Section IV examines techniques to filter this information to extract the road information. Section $\mathrm{V}$ presents a novel technique for extracting significant areas that can be used to form the context area map. Results showing the output of the algorithms using data from real-life mining operations is presented throughout the paper. Section VI provides discussion on the implementation. Finally, the conclusions are presented in Section VII.

\section{A METRIC FOR SAFETY}

Research has found that the $93 \%$ of vehicle accidents have human error as a causal factor [5]. In mining operations, safety managers attempt to minimise this risk of human error by designing protocols for safe operation, and training operators
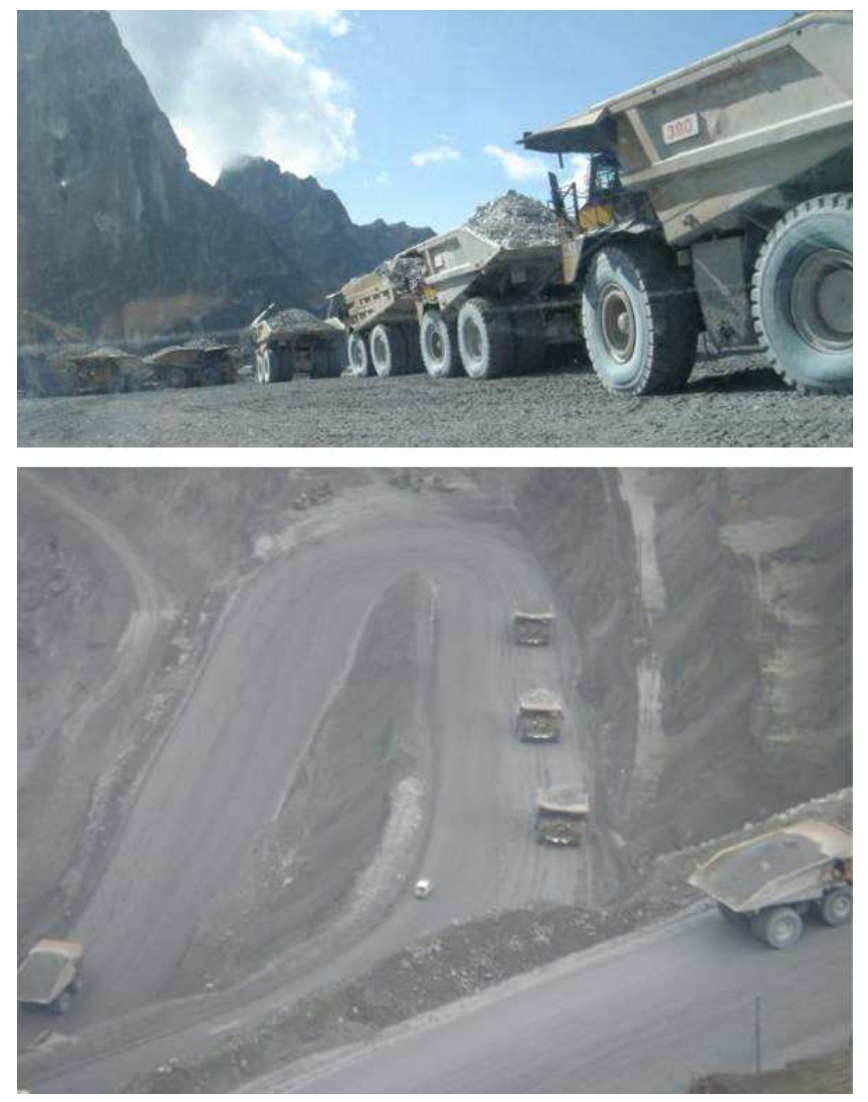

Fig. 2. Two areas of a mine that have different expected meaning for vehicle operators (different context meaning): (a) A dumping area where trucks park close together and (b) A haul road where vehicles must maintain separation of at least $50 \mathrm{~m}$.

to follow these protocols. The safety rules evolve over time as the result of new research, and by analysing the cause of near misses and accidents. Despite the best efforts of mine management to implement rules and protocols for safe vehicle operation, accidents and near misses still occur. Vehicle operators do not normally take unacceptable risks knowningly. High risk situations can occur because of unknown risks caused by factors such as inexperience, adverse weather condition, lack of training, over confidence, boredom, fatigue and many others. Some examples of difficult environmental conditions from a typical mine are shown in Figure 1.

There are many groups currently researching Collision Avoidance Systems (CAS), which attempt to detect vehicles in the nearby area and estimate the risk of a collision (some examples include [11], [6], [7], [8]). Detection of the other vehicles can be either using sensors such as radar, stereo cameras, laser, etc, or using radio communication to broadcast the vehicle state wirelessly. In both cases, a warning is provided to the vehicle operator when the threat is determined to be above a certain threshold based on a variety of algorithms using the available vehicle state information of the nearby vehicles. The main issue with all of these approaches is that there is a trade-off between having a large number of false alarms by providing an early warning of a collision, and providing a warning of a collision at the last possible moment with fewer false alarms when a collision is almost unavoidable. 
If the driver is presented with false alarms, it is likely that they will either not pay attention to the warnings or become annoyed at the unwanted distraction [9]. There are also clear benefits to providing warnings early to allow drivers more time to comprehend the situation [10]. This is a form of reactive feedback, which requires the operator to react fast enough to avoid the imminent collision.

An alternative strategy introduced in this paper is to use preventitive feedback in the case where a high risk scenario is detected, which is the precursor to a collision. The safe rules of operation are well defined for vehicle operations, including rules governing parameters such as minimum separation between vehicles and speed limits. By collecting vehicle state information from nearby vehicles, it is possible to detect breaches of these rules and provide feedback to the operator. This preempts a dangerous situation as the probability of a collision is highest when one (or more) of the safety rules has been breached. The feedback can then be provided to the operators allowing them time to process the information and modify their actions to minimise the risk in the situation.

The main difficulty in measuring safety is that safety is context dependent. The safe rules of operation vary depending on the circumstances of the situation which includes the location, speed, number of nearby vehicles as well as many other factors. The concept of a 'context area' is defined here as the human meaning attached to a specific area that defines the purpose of the area, consequently defining the expectation of behaviour in this area. A example of this can be seen in Figure 2 , where two different context areas in a mine are illustrated. The top image shows a dumping area, where vehicles are queued close together waiting for the opportunity to unload the ore into a crushing machine. The bottom figure shows a haul road, where safety rules dictate that vehicles are not allowed to come within $50 \mathrm{~m}$ of each other. The expectation of behaviour in the loading area is for close vehicle interactions at low speed, whereas in the haul road the vehicles travel at higher speed while maintaining more separation.

It is not feasible to provide feedback and warnings to the vehicle operators unless the context of the situation is taken into account. Presenting unnecessary warnings to an operator reduces the effectiveness of the system as the operator will be much less likely to trust the information, and will also likely be annoyed by the false information [9]. The remainder of this paper focuses on a novel method for extracting information about important context areas in a mine environment using data collected from vehicles in operation. These areas can be named by humans to provide the specific meaning, such as loading area or parking lot. These areas are then used to modify the rules as interpreted by the vehicle system to provide feedback to the operator in the event of a breach of these rules.

\section{Collecting AND Filtering DATA}

The mine environment is highly dynamic, with roads and areas being created and removed regularly. Mining roads are not sealed, and the colour and texture of the roads and traversible areas are often not distinguishable from the surrounding areas.
In many roads or sections of the road there are no marks or berms to indicate the road boundaries. Furthermore the mine is a very dynamic process and roads are changing all the time. This means that image processing and aerial photography are not feasible to be used in the generation of up to date mine maps. The alternative method is to use vehicle position and trajectory information to generate the map.

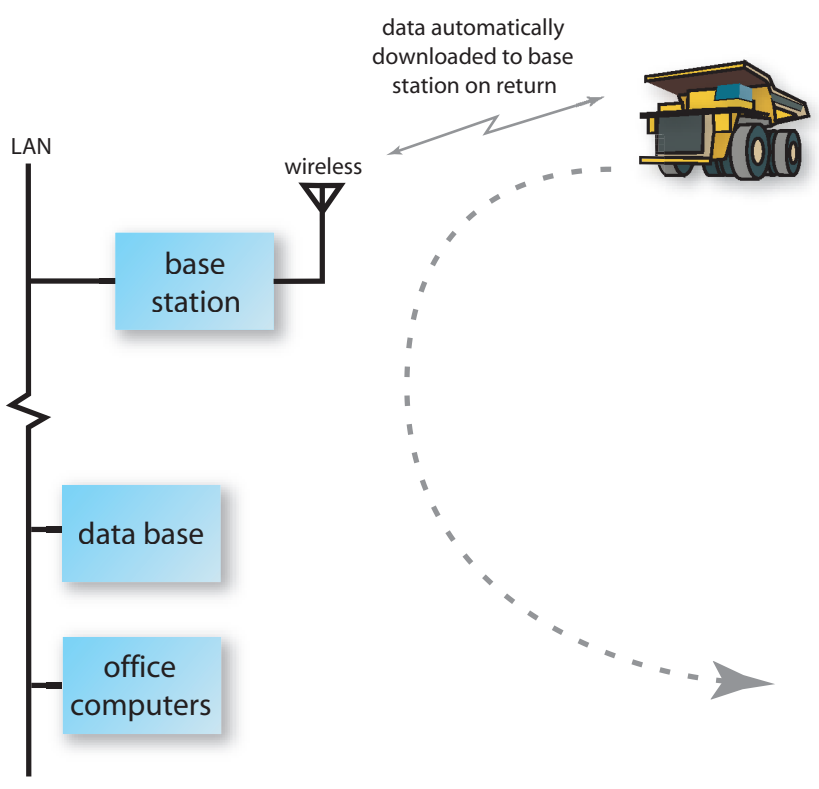

Fig. 3. Data is automatically collected from the vehicles when they are in range of a collection point (either directly or through the mesh).

A system has been designed and installed in several fleets of mining vehicles that can determine the vehicle state information, and to communicate this information with vehicles in the surrounding area using ad-hoc, mesh communication [12]. This system was designed to provide additional situation awareness to the vehicle operators, particularly in mining vehicles which often operate with poor visibility due to evnironmental conditions and visual obstructions from the vehicle cabin. Data is logged within each vehicle, including position information, heading, velocity and other vehicle state information. This data is collected when the vehicles move within range of a data collection point, either directly or though the mesh network of other vehicles. The data is downloaded and stored in a database as described in Figure 3, and can be used for creating mapping information.

The first step in the mapping process is clustering, which is necessary to reduce the amount of data and take the average of potentially noisy position data. Heading information must be considered as a separate dimension to allow the discrimination of position information from vehicles travelling in opposing directions on the same road. Position information from vehicles travelling in opposite directions often overlaps due to the vehicles not staying completely on one side of the road when overtaking or maneuvering around holes, etc. In addition, the position data is sourced from a GPS sensor which can potentially be noisy. These factors mean that the position information can be spread in the axis perpendicular to the direction of the road. To account for this variation, the clusters 
must consider the heading in order to maintain separate lane information for each direction of travel.
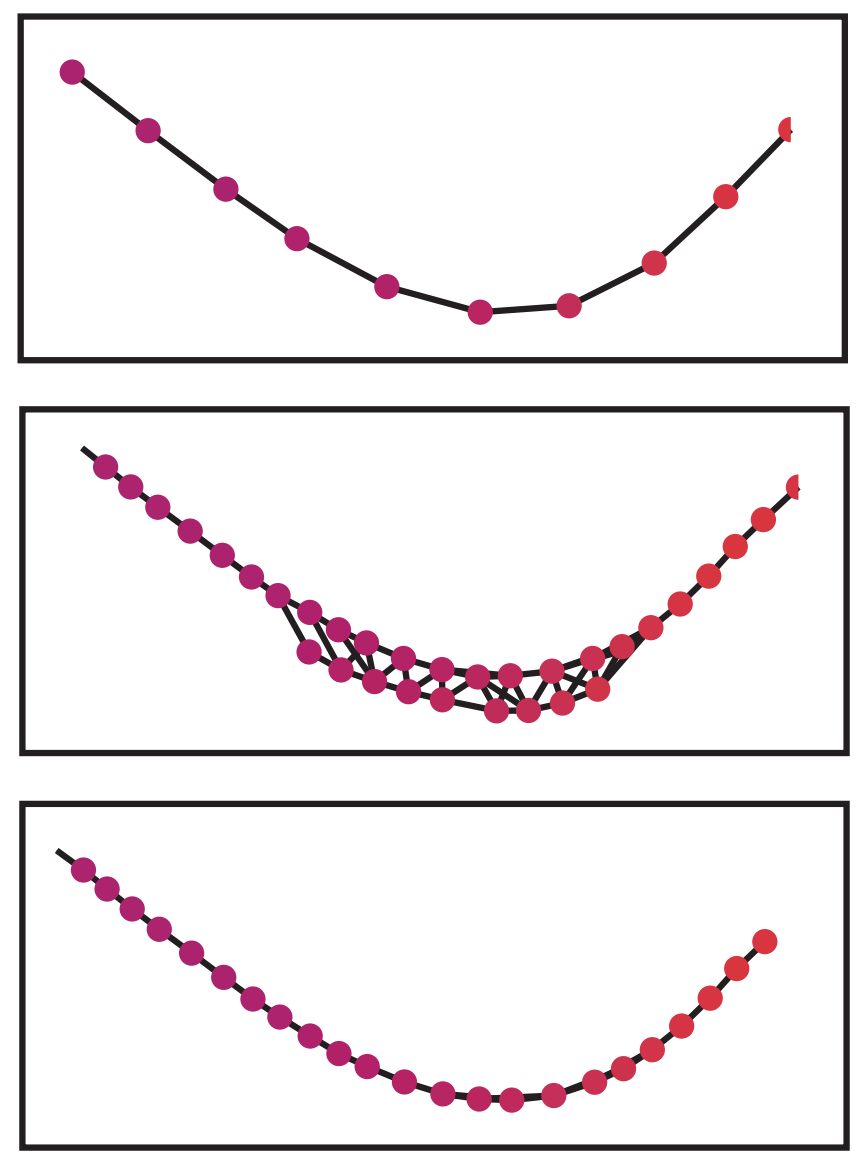

Fig. 4. Different cluster shapes used to group the GPS data (a) shows a curve with cluster size $x=20 \mathrm{~m}, y=20 \mathrm{~m}$, (b) shows cluster size $x=7 \mathrm{~m}, y=7 \mathrm{~m}$, (c) shows cluster size $x=20 \mathrm{~m}, y=7 \mathrm{~m}$.

The road width in mining can vary significantly, ranging from 10 to 30 or more meters in width. To provide an idea of the variety of scale of the vehicles involved, small vehicles can be around 2 meters wide, large vehicles can be over 7 meters in width. Tuning the parameters of a clustering algorithm to account for such variation is difficult, for example if points within a certain radius are clustered together then the radius required would need to be at least 20 to 30 meters to account for the maximum road width. Increasing the collection radius for a cluster means that the clusters are spread further apart along the axis of the road and some fidelity of the road shape is lost, especially around corners and at intersections. A solution to this problem is to change the shape of the area used to collect the position information into a cluster. Figure 4 shows the outcome when using a rectangular shaped cluster area. Points are incorporated into a cluster differently in the $\mathrm{x}$ axis (perpendicular to the direction of travel) compared with the yaxis (following the direction of travel. If points are added into a cluster from a larger range in the $\mathrm{x}$-axis, the fidelity of the road shape is better maintained as seen in Figure 4(c). Figure 4(b) shows the output if the clusters are too small, leading to multiple lines of clusters representing the same section of road.

\section{Extracting RoAds}

Once the point clusters are generated, the next step is to determine the relationship between each cluster. This process involves linking the clusters, and is used to form the road information. A road is defined here as a linked set of clusters where the motion of the vehicle is constrained to a single dimension (direction). This means that each cluster is linked forward to a single cluster, and back to a single cluster. This paper describes three methods of linking clusters that contain position and heading information.

The above definition of roads and areas allows the mine layout to be represented as a directional graph, or digraph. The context areas are used to make the verticies of the graph and the roads form the directional edges that join the verticies. It is possible to form the graph representation by defining a road as the single directional path between two context areas, which can be intersections, or other context areas. This formal representation of this graph structure is presented in Equation 1 , and the graph structure can be seen in the roads and areas shown in Figure 8.

The roads are extracted from the cluster data as places where normal motion is constrained to a single direction of travel. Once these areas are determined, the remaining clusters can be used form areas where the direction of travel is less constrained, where vehicles can move in different directions. These areas represent increased likelihood of an important area with contextual meaning as there is a large potential range of maneuvers in these areas.

$$
\begin{aligned}
\text { Graph of Mine } G & =(V, E) \\
\text { vertices } V & =v_{1} \cup v_{2} \cup \cdots \cup v_{n} \\
& =\text { Set of } n \text { Context Areas } \\
\text { edges } E & =e_{1,2} \cup e_{2,1} \cup \ldots \text { etc } \\
& =\text { Set of Roads }
\end{aligned}
$$

$$
\begin{aligned}
& \text { where } \\
& e_{i, j}=\text { directional link between area } \mathrm{i} \text { and } \mathrm{j}
\end{aligned}
$$

\section{A. Geometric Relationship}

The road information can be determined by considering the geometric relationship between the clusters. Equation 2 introduces a metric that is calculated using a function of the distance between clusters $(\Delta S)$, bearing of the other cluster relative to the test cluster heading $(\phi)$, and the difference in heading between the clusters $(\Delta \theta)$. Each of these measures are weighted using the parameters $\alpha, \beta$ and $\gamma$ and added to give the metric value. These parameters are tuned to suit the application, though there is scope for future work in using feedback to improve the parameter selection within a probabilistic framework.

$$
\text { metric }=\alpha \Delta \mathrm{S}+\beta \phi+\gamma \Delta \theta
$$

The metric is calculated for each cluster relative to the other nearby surrounding clusters. When the metric is within a given 
threshold, the link is created between the clusters. This strategy was successful in most scenarios, with the exception being if two unconnected roads are too close together for the selected distance metric. In this case, there is a tendancy for incorrect links to be made as illustrated in Figure 5(a).

\section{B. Temporal Relationship}

The second approach for linking clusters considered in this paper involves fitting the logged vehicle trajectories to the clusters. A link is strengthened when a vehicle trajectory passes between two clusters. The strength of the link is measured by the number of times a vehicle moves between the clusters. A threshold is used to determine what strength of link is required to create the road, allowing this process to filter out incorrect links. Using vehicle trajectories to generate the links provides a robust linking method that is less likely to fail when different roads are close together. This is because the links are only created between clusters where vehicles have actually traveled. Part (b) of Figure 5 shows the result of the linking process using vehicle trajectories. On the roads, the two approaches for linking clusters produces similar results. The main benefit of the vehicle trajectory approach is seen around intersections.

\section{C. $\alpha$-Shape}

The underlying shape of a set of points can be determined by using the alpha shape technique [13]. This technique uses a circle of radius $\alpha$ to essentially 'scoop' out the area surrounding the border of the points. The process begins by taking a set of points to be included in the shape, and determining the circumcircles that can be created using each pair of points. The boundary of the shape is defined by all the circumcircles that do not contain one of the other points in the set. This means that an $\alpha$-shape can contain holes and concave areas. As $\alpha$ approaches infinity, the shape approaches the convex hull. The value of $\alpha$ was selected to be twice the size of the distance between clusters, meaning that all clusters that should be joined are within the range of the nearest points when creating the circumcircles.

Figure 6(a) shows the set of clusters used to make the $\alpha$-shape. Figure 6(b) shows the resulting $\alpha$-shape, with the circumcircles drawn when they do not contain a different point from the remaining set. It can be seen in this shape that when the roads are far apart, they are considered as separate lines both circumcircles created from the pair of points do not contain another point in the set. When the roads are closer together, the inner circumcircles contain points from the opposing side of the road to create a filled in shape (indicated as a black filled polygon in the image).

To provide the separation between roads, it is possible to consider the heading as an additional dimension to the $\alpha$-shape algorithm. In this case, the test of other points that lie within the circumcircle excludes points that have a heading difference that is larger than a particular threshold. This means that the clusters that belong to the opposing side of the road are not included, and the $\alpha$-shape can represent the different sides of the roads as shown in Figure 6(c).
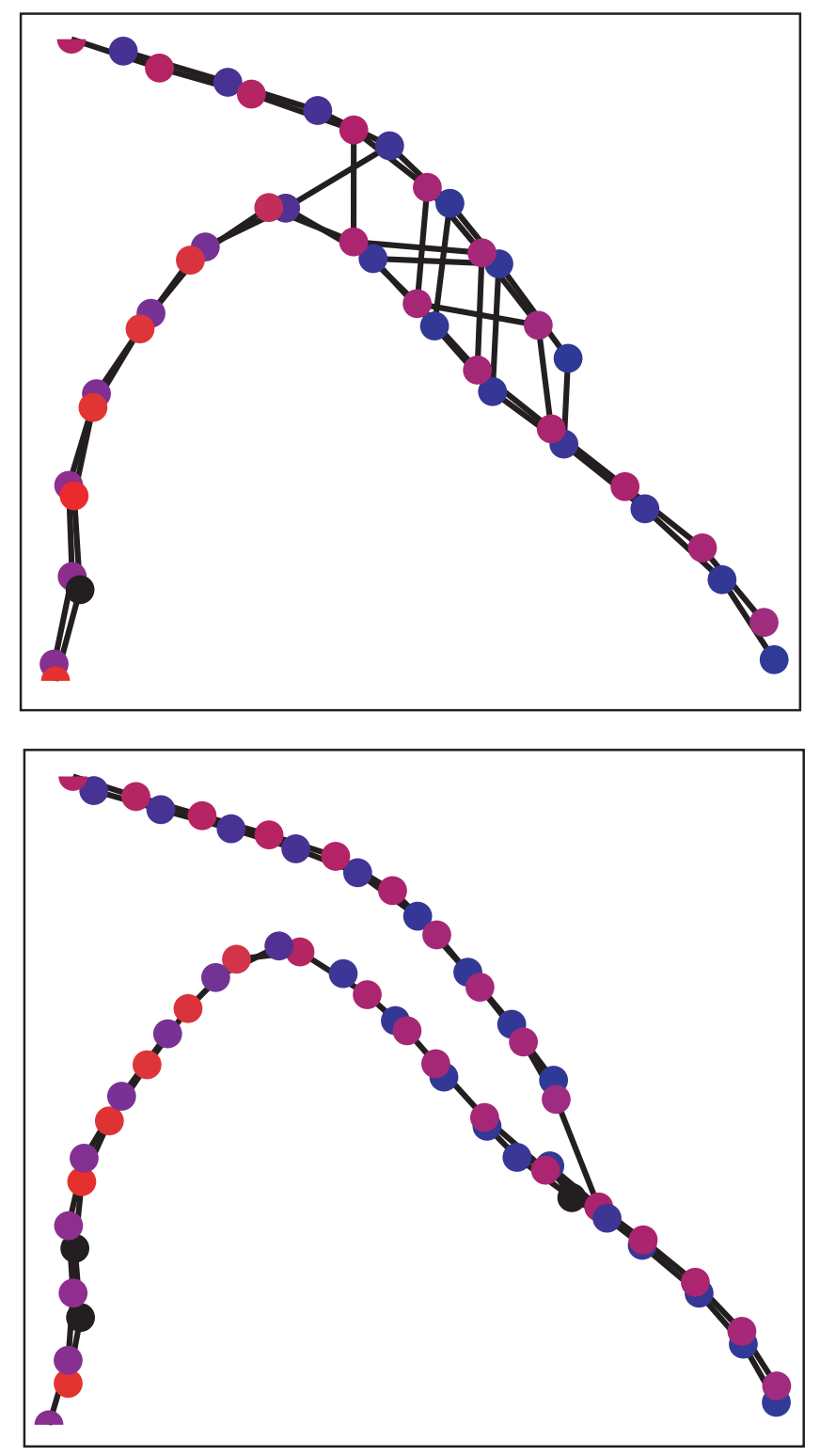

Fig. 5. Shows a potential issue with geometric based linking of clusters (a) geometric linking where incorrect links are made to a nearby road (b) linking using the actual vehicle trajectories solves this problem.

\section{Context Area Shapes}

Section IV described the process of determining the location of roads within the mine where the vehicle motion is constrained to a single direction. The remaining clusters then indicate the areas where the vehicle movement is less constrained and more complex than on a road. The additional complexity generally means there is a greater chance of a misunderstanding between two nearby vehicles, and that the area has some additional meaning to the vehicle operators. These areas include intersections and other open areas such as parking lots, loading areas, dumping areas, refuelling bays, etc. These areas are of interest as the expectation of behaviour of other nearby vehicles is likely to be different to being on a road. In a parking lot for example, there is an expectation that a vehicle is likely to drive slower and maneuver into a parking space at any moment. 


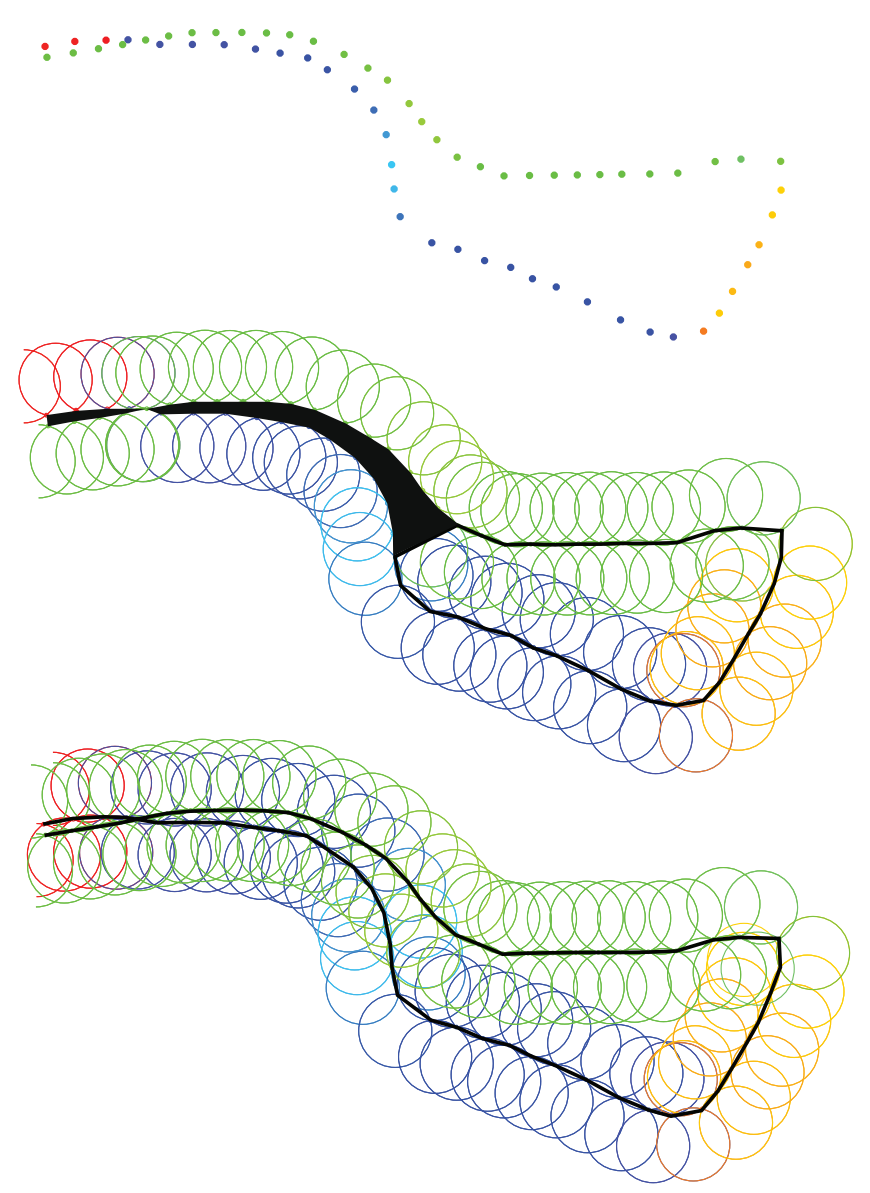

Fig. 6. Using $\alpha$-shapes to extract road information from a set of clusters. (a) shows the clusters only, (b) shows the $\alpha$-shape with the circumcircles not containing another point from the set drawn and (c) shows the $\alpha$-shape created when the circumcircle test ignores the points where the difference in heading is greater than a threshold (in this example, 90 degrees).

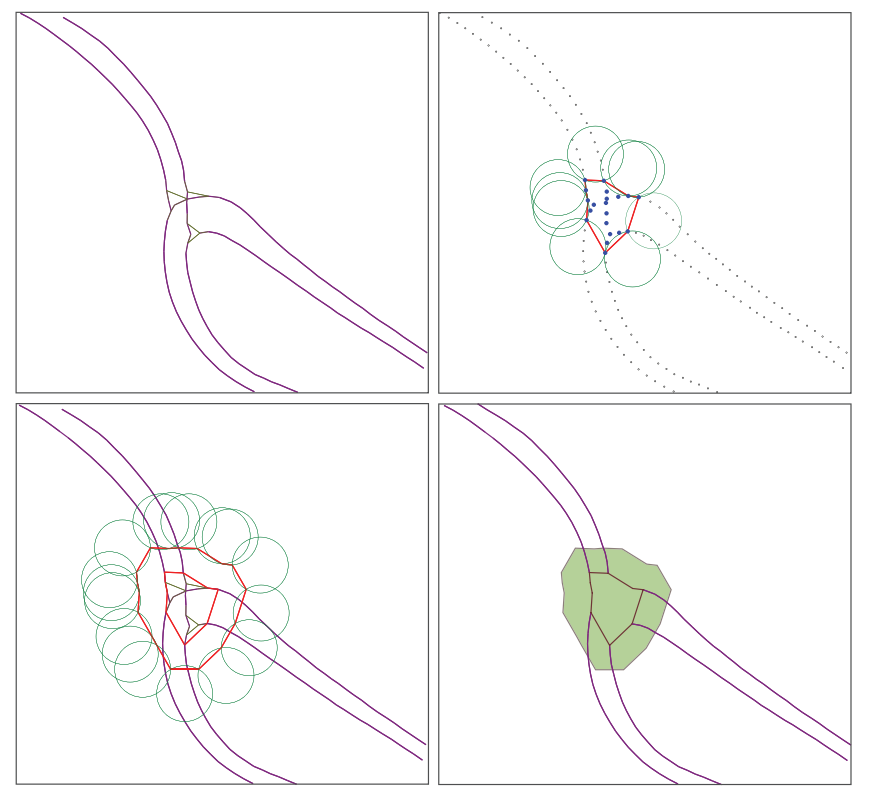

Fig. 7. Using $\alpha$-shapes to build an intersection. (a - top left) shows the roads created by the process described in Section IV-C. (b - top right) shows the clusters not associated with a road used to make a normal $\alpha$-shape. (c bottom left) The points in the same intersection are dialated using a hexagon shape of points. (d - bottom right) The final context area is shown.

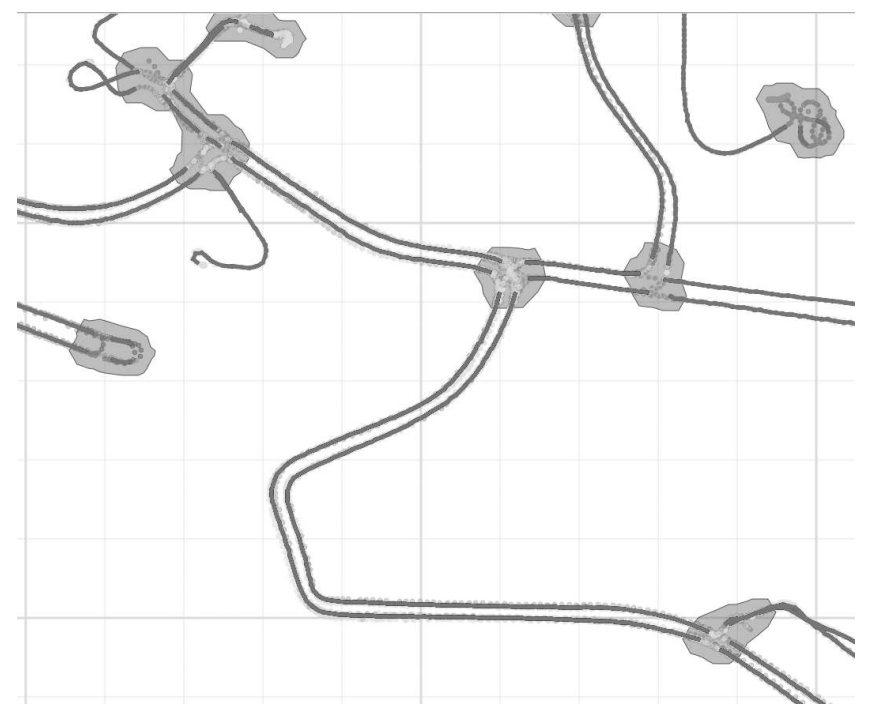

Fig. 8. The map shows the output of the algorithm to generate the roads and determine important areas. The roads are represented by black lines, the areas by filled polygons. Position information used to generate the map is plotted behind the roads.

The context area is defined by creating $\alpha$-shapes out of the clusters that are not already affiliated with a road. Figure 7(a) shows a typical intersection in a mine, with the roads overlaid. At the intersection, the roads finish because they link forward to more than one other cluster at the junctions. Figure 7(b) shows the clusters only, the clusters not associated with a road form a shape using the $\alpha$-shape algorithm.

At this point, the shape represents the intersection, but too tightly because each cluster represents only the average point from the data collected in the cluster shape (described in Section III). The area is better represented by dialating the clusters in the context area and using the expanded set of points to make a larger shape. The clusters are dialated by the normal distance between clusters, which was empirically set to 10 meters. Six points were taken at this radius around each point in the context area making a hexagon shape, with the intention that this would be sufficient to represent the expanded shape. The resulting points and $\alpha$ shape can be seen in Figure 7(c). The final context area shape can be seen in 7(d).

Figure 8 shows the combination of many context areas and roads that was created from real data collected from a mining operation. The roads are shown as black lines, and the areas are represented by filled polygons. The GPS information that was used to create the roads is plotted behind the roads to show how well the context areas cover the area used by the vehicles.

\section{EXPERIMENTAL RESULTS}

Figure 9 shows the roads and context areas created using the algorithms described in this paper overlaid on an aerial photograph of the mine. The high risk areas in this image are the intersections, especially the large, four-way intersection in the center of the image. This intersection is a potentially high risk environment as there could be vehicle approaching from any of four directions, and the intended destination of each 


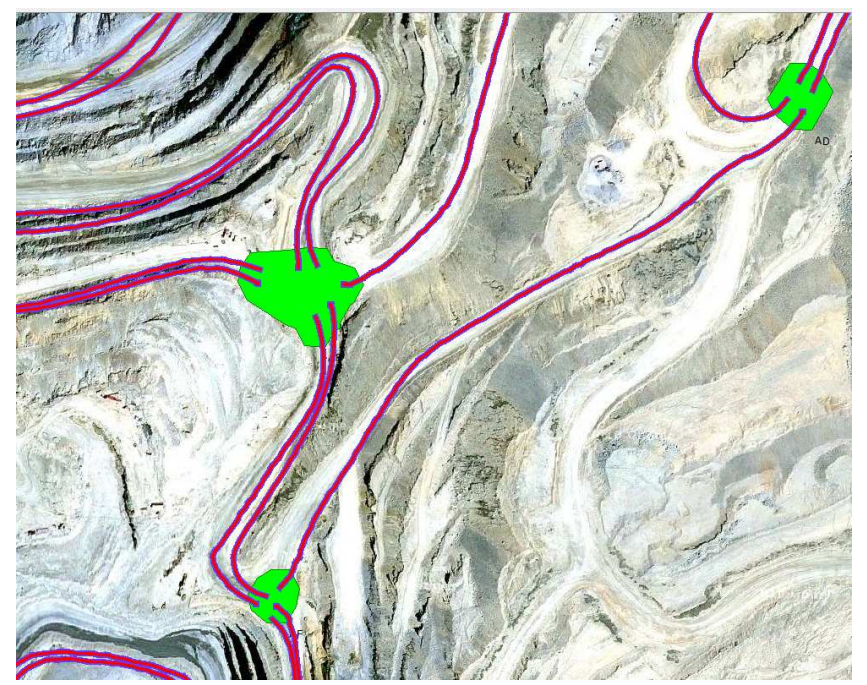

Fig. 9. Roads and context areas are overlayed on a aerial image of the mine site. The important context areas in this image are the intersections, particularly the large, four-way intersection in the center of the image.

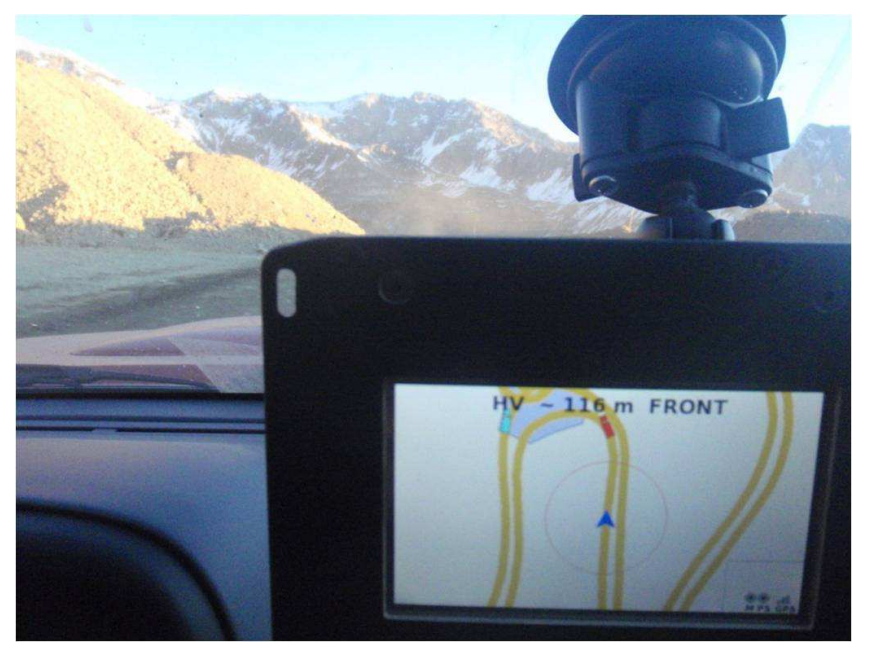

Fig. 10. The operator interface provides situation awareness information to the driver. There are two vehicles displayed on the screen that outside the operator's line of sight. An area of interest is shown at the top of the screen representing a large intersection.

vehicle is unknown to the other vehicles. These roads and context areas are then automatically distributed to the fleet of vehicles and are used for the operator interface.

Figure 8 shows the quality of the areas generated from the algorithms introduced in this paper. The correctness of the areas can only be determined by the mine personnel who are familiar with the roads and layout of the mine, and is essential before the maps can be used in the operator interface. The feedback from the mine personnel is that the algorithm provides a good representation of the context areas in the majority of cases. This subjective analysis is not yet quantified. Future research in this area will examine metrics to automatically determine the quality of the generated maps. Two main factors were determined that impact the quality of the created map. In the event of poor GPS reception, it is possible that the noise in the position estimate can cause errors in the context area shape. In addition, data collected from vehicles performing maneuvers that are uncharacteristic given their location presents another potential cause of incorrectly generated context areas. An example of this is if a vehicle performs a u-turn maneuver on a section of haul road (which they are not supposed to do), this area could potentially be incorrectly interpreted as being a context area. Detecting and filtering out these cases is an area of future work.

Figure 11 shows the operator interface in the vehicle cabin designed to improve the operator's situation awareness. At a glance, it is possible for the operator to determine that they are in a context, or important area (labelled in this case as a Crusher Area). The nearby vehicles are displayed, and the distance icon accompanied by a short sound indicates that the distance is too close to the vehicle in front based on the rules determined for that specific context area. When the rules implemented for this specific context area are breached, the icons shown on the bottom of the image are displayed along with the appropriate sound warning.

Figure 10 shows the interface working in a mine site, providing situation awareness to the operator. The screen shows two vehicles in front, neither of which can be seen directly from the vehicle. The coloured area at the top of the display indicates that there is an important area (in this case an intersection) around the next corner which is not in a line of sight from the vehicle. This simplified representation of the environment allows the operator at a glance to improve their understanding of the current situation.

The current vehicle position relative to the roads and areas on the map provide an important piece of information to the vehicle operator. In poor weather conditions, it can be difficult to determine the vehicle location relative to the intersections and other areas, especially for an inexperienced driver. Providing the map with hilighted intersections and areas to the operator should improve the awareness of the current location.

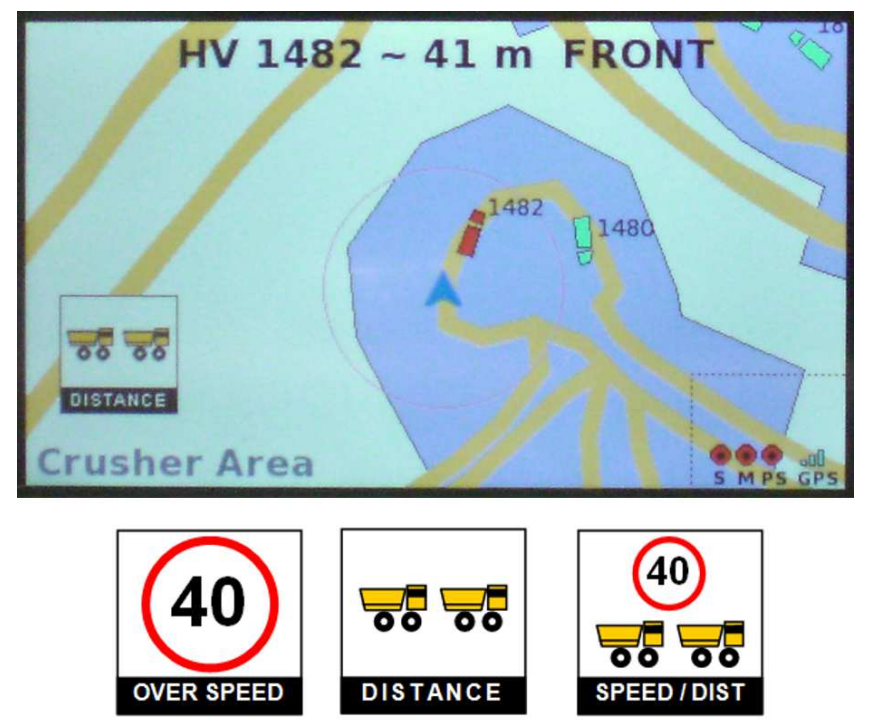

Fig. 11. Display implementation currently installed in mining vehicles. The context area name can be seen in the bottom left of screen. Several warning icons representing breaches of the rules are presented. 


\section{CONCLUSION}

This paper introduced a method of determining risk using context based rules. A method for generating up to date road maps including the evaluation of context areas was presented using vehicle trajectory data collected from real-life mining vehicle fleets. The context area was generated using a process of clustering raw data, extracting road information from the relationships between the clusters and finally using the remaining clusters to generate an $\alpha$-shape representing a potential area of interest. The context areas can be provided with a label by a human to make it more understandable to the vehicle operator. There is scope for future work in this area to automate this process by probabilistically determining the semantic meaning of each area.

Without the context information, it is not possible to measure risk based on rules that vary depending on location. It is necessary to consider the context of the situation before providing potentially incorrect feedback to the vehicle operator. Unnecessary or false alarms are detrimental to the trust and usefulness of the system, and can potentially be distracting and annoying. This work can be extended outside the field of mining operations. There are many parallels with the operation of mining vehicles and passenger vehicles. Mining operations are generally present a much more difficult environment with unsealed roads, large range of vehicle sizes and 24 hour operation.

\section{REFERENCES}

[1] E. Nebot, J. Guivant, and S. Worrall, "Haul truck alignment monitoring and operator warning system," Journal of Field Robotics, vol. 23, no. 2, pp. 141-161, March 2006.

[2] S. Worrall and E. Nebot, "Using non-parametric filters and sparse observations to localise a fleet of mining vehicles," in IEEE International Conference on Robotics and Automation, 2007, April 2007, pp. 509-516.
[3] S. Worrall and E. Nebot, "A probabilistic method for detecting impending vehicle interactions," in Robotics and Automation, 2008. ICRA 2008. IEEE International Conference on, 19-23 2008, pp. 1787 -1791.

[4] G. Agamennoni, J. Nieto, and E. Nebot, "Mining gps data for extracting significant places," in ICRA'09: Proceedings of the 2009 IEEE international conference on Robotics and Automation. Piscataway, NJ, USA: IEEE Press, 2009, pp. 1860-1867.

[5] M. S. Sanders and B. E. Shaw, "Research to determine the contribution of system factors in the occurrence of underground injury accidents," USBM OFR 26-89, NTIS PB 89-219638/AS, 165 pp., Tech. Rep., 1988.

[6] Y.-J. Chen, C.-C. Chen, S.-N. Wang, H.-E. Lin, and R. Hsu, "Gpsensecar - a collision avoidance support system using real-time gps data in a mobile vehicular network," in Systems and Networks Communication, 2006. ICSNC '06. International Conference on, 2006, pp. 71-76.

[7] J. Huang and H.-S. Tan, "Impact of communication reliability on a cooperative collision warning system," Intelligent Transportation Systems Conference, 2007. ITSC 2007. IEEE, pp. 355-360, September 2007.

[8] O. Gietelink, D. Verburg, K. Labibes, and A. Oostendorp, "Pre-crash system validation with prescan and vehil," Intelligent Vehicles Symposium, 2004 IEEE, pp. 913-918, June 2004.

[9] B. Donmez, L. N. Boyle, J. D. Lee, and D. V. McGehee, "Drivers' attitudes toward imperfect distraction mitigation strategies," Transportation Research Part F: Traffic Psychology and Behaviour, vol. 9, no. 6, pp. 387-398, Nov. 2006. [Online]. Available: www.sciencedirect.com/science/article/B6VN8-4JJ2BVX1/2/a091f0f1acc2864af9b5d9b66b33681e

[10] J. D. Lee, M. L. Ries, D. V. McGehee, and T. L. Brown, "Can collision warning systems mitigate distraction due to in-vehicle devices?" NHTSA, Tech. Rep., May 2000. [Online]. Available: wwwnrd.nhtsa.dot.gov/departments/nrd-13/driver-distraction/PDF/31.PDF

[11] S. Kodagoda, S. Sehestedt, A. Alempijevic, Z. Zhang, A. Donikian, and G. Dissanayake. Towards an enhanced driver situation awareness system. Industrial and Information Systems, 2007. ICIIS 2007. International Conference on, pages 295-300, Aug. 2007.

[12] S. Worrall, D. Orchansky, and E. Nebot, "Improving situation awareness with a high integrity proximity system," Australasian Mine Safety Journal, vol. 2, pp. 94-97, 2009.

[13] H. Edelsbrunner and E. Mücke, "Three-dimensional alpha shapes," in Proceedings of the 1992 workshop on Volume visualization. ACM, 1992 , p. 82. 